HEXACO Traits, Big Five Traits, and COVID-19

Chris C. Martin

Coulter Department of Biomedical Engineering

Georgia Institute of Technology, Atlanta, GA

This is an unedited manuscript that may be submitted for publication.

Author Note

This research was supported by the National Science Foundation under IUSE/PFE:RED grant number 1730262. I have no conflict of interest to declare.

I thank Joseph M. Le Doux, Wendy Newstetter, Maysam Nezafati, Johannes Leisen, and Edward Botchwey for helping with the execution of this study.

This paper uses 1.5-line spacing to conserve paper.

Corresponding Author: Chris C. Martin, 313 Ferst Dr., Suite 3118, Atlanta GA 303320535. chris.martin@bme.gatech.edu. 


\begin{abstract}
Trait research on health has primarily focused on chronic diseases, and those findings may not generalize to acute infectious diseases. In the current study, I analyze the correlations between personality traits and attitudes toward COVID-19. Data were collected at the onset of the U.S. epidemic, when COVID-19 was salient to the public. Questionnaires were administered to 172 undergraduates at a public university. These questionnaires measured seven attitudes toward COVID-19, personal risk, and public health. HEXACO trait data had already been collected at this time from all participants, and Big Five data had been collected from a subset of 100 participants. The findings suggest that there are weak-to-moderate correlations but no strong correlations between Big Five traits, HEXACO traits, and attitudes. Conscientiousness was associated with greater trust, and openness with less trust in U.S. and European governments' preparedness for future epidemics. Openness was also associated with less fear of getting infected after being exposed to a person who had or was exposed to COVID-19. The generalizability of these results is low, but this information may be useful for setting priors in Bayesian statistics for future studies. The findings also pertain to the convergent validity of HEXACO and Big Five trait models.
\end{abstract}

Keywords: COVID-19, coronavirus, HEXACO, Big Five, personality traits, health, infectious diseases 


\section{HEXACO Traits, Big Five Traits, and COVID-19}

Personality traits predict health and illness, but most trait studies have focused on chronic diseases rather than severe and acute diseases (Turiano et al., 2012). This bias toward chronic diseases is likely a reflection of the fact that the public health response to severe acute diseases is often swift, shortening the timespan in which the disease is salient to citizens and relevant to psychologists. At the time of the COVID-19 pandemic, I was conducting panel studies of over 200 students and had already collected trait data from the sample using long forms with better internal consistency than the short forms typically used in public surveys. I therefore used this opportunity to conduct exploratory research on personality traits and COVID-19. This research was not conducted with a representative sample of adults, but the findings may be useful for future research with such samples. There was a modest degree of socioeconomic and ethnic diversity in the sample.

The COVID-related attitudes here span seven topics: travel, fear of infection, perceived governmental preparedness, perceived severity of the disease, suspicion toward exposed persons, and interest in vaccines. The study was partially modeled after the Emory University Ebola survey, designed by Ralph DiClemente and Nihari Patel, in which these topics were among those included. The background for the design of the Ebola study is described elsewhere (Painter, DiClemente, \& von Fricken, 2017). Although the Ebola study focused on public health, some findings from the Ebola survey have been published in the psychology literature (Martin, 2020).

Both Big Five traits and HEXACO traits are examined here, but the sample for Big Five traits is a subset $(n=100)$ of the larger sample $(n=172)$. The inclusion of both trait models complements studies on traits and COVID-19 that only use one model (Abdelrahman, 2020; Flesia, Fietta, Colicino, Segatto, \& Monaro, 2020; Kroencke, Geukes, Utesch, Kuper, \& Back, 2020).

The current study was exploratory and there are no hypotheses to report. The data were analyzed using simple bivariate analyses. Because the health attitudes were measured with single questions with short Likert scales, I used polychoric and polyserial correlations rather than Pearson correlations to model associations. The valence of correlations is typically the same regardless of correlation type, but the magnitude can differ. Data were collected in the first week of April 2020, when South Korea, Italy, China, and Iran were bearing the brunt of the pandemic. 
Shutdowns had been mandated in many U.S. states and localities at the time, and the U.S. case count was approximately 30,000.

\section{Method}

\section{Participants}

Participants were students enrolled in two undergraduate classes at the Georgia Institute of Technology. Most participants were majoring in biomedical engineering and both classes were required for students in this major. The first class was a prerequisite for the second class, and therefore no participants were enrolled in both classes at the same time. Participants had begun to take part (or had finished taking part) in four- and five-wave panel studies at the time of the COVID-19 pandemic. COVID questions were added to a wave of the panel study. In the current paper, data from 172 participants are used in HEXACO analyses in this paper (112 participants in the first class and 60 participants in the second class). Approximately $70 \%$ of participants were female, $45 \%$ were White, and 30\% were Asian. The mean age was $19.3(\mathrm{SD}=$ 1.7) and $95 \%$ of participants were in the 18-21 range. The characteristics of the sample are listed in Table 1, which includes a breakdown of how many international flights the participant took in the last 12 months, which is pertinent to an international pandemic.

Trait data were collected at the beginning of the panel studies in an initial survey that either one or two weeks before the first wave. Big Five trait data were only collected in the first class, and the sample size for corresponding analyses is 100 . Two participants had traveled to South Korea, China, Iran, or Italy in the two months preceding the administration of the COVID questions.

Table 1. Descriptive statistics and pairwise correlations for Study 1.

\begin{tabular}{lrr}
\hline Factor & Percent & $\mathrm{N}$ \\
\hline Gender & & \\
Male & 30.2 & 52 \\
Female & 68.6 & 118 \\
Other & 0.6 & 1 \\
Missing & 0.6 & 1
\end{tabular}




\begin{tabular}{|c|c|c|}
\hline \multicolumn{3}{|l|}{ Race/Ethnicity } \\
\hline White & 46.5 & 80 \\
\hline Hispanic & 8.7 & 15 \\
\hline Black & 7.0 & 12 \\
\hline Asian & 29.7 & 51 \\
\hline Asian-White & 3.5 & 6 \\
\hline M. Eastern & 2.9 & 5 \\
\hline Other & 1.2 & 2 \\
\hline Missing & 0.6 & 1 \\
\hline \multicolumn{3}{|l|}{ Disability } \\
\hline No & 92.4 & 159 \\
\hline Yes & 7.0 & 12 \\
\hline Missing & 0.6 & 1 \\
\hline \multicolumn{3}{|l|}{ International } \\
\hline No & 93.6 & 161 \\
\hline Yes & 5.2 & 9 \\
\hline Missing & 1.2 & 2 \\
\hline \multicolumn{3}{|l|}{ Mother's Educ. } \\
\hline 8th grade or less & 2.3 & 4 \\
\hline High school & 6.4 & 11 \\
\hline Some college & 7.0 & 12 \\
\hline Associate's & 6.4 & 11 \\
\hline Bachelor's & 41.3 & 71 \\
\hline Graduate deg. & 36.0 & 62 \\
\hline Missing & 0.6 & 1 \\
\hline \multicolumn{3}{|l|}{ Father's Educ. } \\
\hline 8th grade or less & 2.9 & 5 \\
\hline 9th-12th grade & 1.2 & 2 \\
\hline High school & 6.4 & 11 \\
\hline Some college & 5.8 & 10 \\
\hline Associate's & 2.9 & 5 \\
\hline Bachelor's & 29.1 & 50 \\
\hline Graduate deg. & 48.8 & 84 \\
\hline Don’t know & 2.3 & 4 \\
\hline Missing & 0.6 & 1 \\
\hline \multicolumn{3}{|l|}{ Recent Intl. Flights } \\
\hline 0 & 61.6 & 106 \\
\hline $1-2$ & 23.3 & 40 \\
\hline $3-4$ & 9.3 & 16 \\
\hline $5-6$ & 3.5 & 6 \\
\hline $7+$ & 2.3 & 4 \\
\hline Total & & 172 \\
\hline
\end{tabular}

Note. Recent Intl. Flights refers to the number of international flights taken by the respondent in the last 12 months. Mothers' and father educational attainment refer to attainment by "mother or maternal equivalent" and "father or paternal equivalent" respectively. 


\section{Procedure}

At the beginning of the semester, a researcher visited a course session to explain the purpose of the surveys and notified students that they would receive email invitations and reminder emails. The procedures were declared exempt from review by the Institutional Review Board.

Data were collected using Qualtrics and email invitations were scheduled through Qualtrics. A reminder email was sent to students who did not respond to the initial invitation for every survey. The email invitation contained a link to the first page of the survey, which contained the consent form. In cases where students could get course credit for participation, the consent form described the credit and explained an alternate method of getting equivalent credit, which was to submit a short summary of a research article or blog post.

Each wave was divided into question blocks. The COVID-19 block was used in a survey in the first week of April 2020.

In the first class, it was inserted into the third wave of the ongoing four-wave study along with other social psychological questions. Respondents were informed in advance by e-mail that some health questions had been added to the third wave. If they desired, respondents could opt out of the COVID questions by leaving them blank. Students were compensated for participating in this study.

In the second class, the five waves of data collection were complete. The COVID-19 block was therefore inserted into an extra sixth wave. The five-wave survey had compensation attached to it. This extra wave only contained COVID-19 questions and participants received no compensation for completing it. Participants were invited to take this extra wave because of its value to the scientific community.

De-identified data are available on the OSF website at https://osf.io/uh8q3/. These data only contain partial demographic information to protect anonymity.

\section{Measures}

Demographic Variables. Demographic questions were modeled after items in the Healthy Minds 2015-16 Study (Healthy Minds Network, 2016).

Gender was measured with a single item with six options: "male," "female," "trans male/trans man," "trans female/trans woman," "genderqueer/gender non-conforming," and 
"other." Answers were recoded into a three-level variable: 1 (male), 2 (female) and 3 (other). This recoding was used because of the small number of responses in the last four categories.

Race and ethnicity (henceforth race) was measured with a single question where students could select multiple options simultaneously. The recoding here follows Martin (2019). A participant was coded as (a) White if they exclusively selected "White," (b) Hispanic if they selected "Hispanic" and up to two other options, (c) Black if they exclusively selected "African American/Black," (d) Asian if they exclusively selected "Asian," (e) Asian-White biracial if they exclusively selected "White" and "Asian," (f) Middle Eastern if they exclusively selected "Middle Eastern, Arab, or Arab American," (g) Other if they selected any other single option, e.g., "American Indian or Alaskan Native," "Native Hawaiian or Pacific Islander, "Other" or a set of options not specified above, and (h) Unknown if they selected none of the options.

In the current paper, gender, race, and ethnicity are only used to describe sample characteristics.

Personality Traits. The 100-item revised HEXACO personality inventory (HEXACOPI-R) was used to measure HEXACO traits (Lee \& Ashton, 2018) It was embedded in the initial survey of each class, which preceded the panel waves. Of the 258 students who participated in the larger study, 225 partially or fully filled out the HEXACO-PI-R. The BFI-2 was used to measure to measure Big Five personality traits in the second class only (Soto \& John, 2017). Participants were asked to take this survey without supervision and report their final Big Five scores in the first survey wave. Of the 165 students who participated in the larger study, 117 entered their BFI-2 scores. However, students who filled traits surveys did not necessarily fill the COVID-19 question block and thus sample sizes here are smaller.

COVID-19. Attitudes pertaining to COVID-19 and health policy were measured using 14 (or 16?) questions, each of which was derived from an Ebola survey (Martin, 2020; Painter et al., 2017). There were two questions each under each of these topics: travel, fear of infection, perceived governmental preparedness, perceived severity of the disease, suspicion toward exposed persons, and interest in vaccines. The full text of all questions is in the appendix. Although there were two questions per topic, permitting the constructing of a two-item scale for each topic, I chose to present the results of each question individually. To be consistent with the Ebola survey, items were measured on 4-point Likert scales, with one exception. The response options varied by question (see appendix), but for most questions, they ranged from strongly 
disagree to strongly agree. The exception to the 4-point scale was an item on how much a participant would pay for a vaccine, assuming a vaccine existed, and had a 6-item scale ranging from 1 ( $\$ 0$ [I would not pay for a COVID vaccine]) to 6 (More than \$200).

\section{Results}

Descriptive statistics of traits are in Table 2. No skewness and kurtosis levels suggest sample abnormality. Henceforth, the term openness encompasses openness to experience (HEXACO) and open-mindedness (B5), and the term emotionality encompasses emotionality (HEXACO) and negative emotionality (B5) unless otherwise specified.

Table 2. Descriptive Statistics of Personality Traits

\begin{tabular}{lcccccc}
\hline & $\mathrm{M}$ & Median & $\mathrm{SD}$ & $\mathrm{N}$ & Kurtosis & Skewness \\
\hline Honesty-Humility (HEXACO) & 3.5 & 3.4 & 0.6 & 172 & 2.8 & -0.2 \\
Emotionality (HEXACO) & 3.5 & 3.4 & 0.6 & 172 & 2.5 & -0.3 \\
Extraversion (HEXACO) & 3.3 & 3.3 & 0.6 & 172 & 2.8 & -0.2 \\
Agreeableness (HEXACO) & 3.1 & 3.2 & 0.6 & 172 & 3.0 & $<0.05$ \\
Conscientiousness (HEXACO) & 3.7 & 3.8 & 0.6 & 172 & 2.7 & -0.2 \\
Openness (HEXACO) & 3.5 & 3.5 & 0.6 & 172 & 2.6 & -0.1 \\
Extraversion (B5) & 3.4 & 3.4 & 0.7 & 99 & 2.9 & -0.2 \\
Neg. Emotionality (B5) & 3.0 & 2.9 & 0.8 & 99 & 2.7 & $<0.05$ \\
Conscientiousness (B5) & 3.8 & 3.8 & 0.7 & 99 & 2.1 & -0.1 \\
Agreeableness (B5) & 3.8 & 3.9 & 0.6 & 99 & 3.1 & -0.4 \\
Openness (B5) & 3.8 & 3.8 & 0.5 & 99 & 2.9 & -0.3 \\
\hline
\end{tabular}

Note. In Stata, the kurtosis of a normal distribution is 3 .

Table 3. Descriptive Statistics of COVID-19 Attitudes ( $\mathrm{N}=172)$

\begin{tabular}{lcccccc}
\hline & M & Median & SD & Kurtosis & Skewness & Range \\
\hline U.S. Preparedness & 1.6 & 1 & 0.7 & 4.2 & 1.2 & $1-4$ \\
European Preparedness & 1.9 & 2 & 0.8 & 2.8 & 0.5 & $1-4$ \\
Risk of infection & 2.4 & 2 & 0.8 & 2.6 & 0.2 & $1-4$ \\
Good possibility of infection & 2.4 & 2 & 0.8 & 2.5 & $<0.05$ & $1-4$ \\
COVID is severe & 3.2 & 3 & 0.7 & 2.7 & -0.4 & $1-4$ \\
COVID deadlier than measles & 2.5 & 2 & 0.8 & 2.6 & 0.2 & $1-4$ \\
Hazardous to share class/workspace & 2.7 & 3 & 0.9 & 2.2 & -0.1 & $1-4$ \\
Hazardous to share airplane row & 2.6 & 3 & 0.8 & 2.6 & $<0.05$ & $1-4$ \\
Likelihood of getting vaccine & 3.6 & 4 & 0.8 & 4.8 & -1.7 & $1-4$ \\
Payment for vaccine (1-6 scale) & 0.9 & 1 & 0.3 & 9.6 & -2.9 & $1-6$ \\
\hline NOte. In Stata the kutosis of & & & & &
\end{tabular}

Note. In Stata, the kurtosis of a normal distribution is 3. 
Descriptive statistics of COVID attitudes are in Table 3. Participants rated both the U.S. and European governments as somewhat unprepared for future outbreaks. The median rating for the U.S. was lower than Europe, and it was also the scale minimum, suggesting little confidence in the U.S. government. Participants were less concerned with personally being infected but they perceived the disease itself as quite severe. They were also quite wary of sharing a classroom or workspace with a person who had been previously diagnosed with COVID-19 and they thought they would likely get infected with COVID-19 if they sat next to someone on an airplane who was from an affected country.

Participants were very likely to get vaccinated if a vaccine were available. The median rating on this item was the scale maximum. The distribution of answers for how much they would pay for a vaccine was apparently right censored. The modal answer was $\$ 1-\$ 50$, chosen by $23 \%$ of respondents. The percentage decreases as the price increased: $22 \%$ chose $\$ 51-\$ 100$, $18 \%$ chose $\$ 101-\$ 150$, and $8 \%$ chose $\$ 151-\$ 200$, indicative of price elasticity (cf. Kondo, Hoshi, \& Okubo, 2009). However, 19\% chose “more than \$200," suggesting that the right tail was censored.

Polychoric correlations between attitudes are in Table 4. As anticipated, there were strong correlations between variables that were related by topic. These correlations are in bold. There were small-to-moderate correlations between perceived possibility of personal infection and the two vaccine variables - likelihood of getting a vaccine and willingness to pay for a vaccine - a pattern that is self-explanatory.

Respondents who were willing to pay more money for a vaccine were surprisingly less likely to estimate that COVID was deadlier than the measles and that it was hazardous to share spaces with people who may be infected. It is plausible that wealthy respondents were more confident in their ability to withstand risk or less aware of contextual factors, but also more willing to pay large amounts for the vaccine (Kraus, Piff, Mendoza-Denton, Rheinschmidt, \& Keltner, 2012). The polyserial correlation between socioeconomic status (derived from four items) and payment for vaccine was .28 , which suggests that maximum payment for a vaccine may partially be explained by household income.

Table 4. Polychoric correlations between COVID-19 attitudes 


\begin{tabular}{lccccccccc}
\hline & 1 & 2 & 3 & 4 & 5 & 6 & 7 & 8 & 9 \\
\hline 1. U.S. Preparedness & 1.00 & & & & & & & & \\
2. European Preparedness & $\mathbf{0 . 6 3}$ & & & & & & & & \\
3. Risk of infection & -0.02 & -0.03 & & & & & & & \\
4. Good possibility of infection & -0.02 & 0.03 & $\mathbf{0 . 7 0}$ & & & & & & \\
5. COVID is severe & -0.15 & -0.02 & -0.07 & -0.10 & & & & & \\
6. COVID deadlier than measles & -0.13 & 0.06 & -0.07 & -0.10 & $\mathbf{0 . 5 4}$ & & & & \\
7. Hazard: share class/workspace & -0.16 & 0.00 & 0.06 & -0.12 & 0.20 & 0.26 & & & \\
8. Hazard: share airplane row & -0.10 & -0.05 & 0.10 & 0.12 & 0.14 & 0.14 & $\mathbf{0 . 4 4}$ & & \\
9. Likelihood of getting vaccine & -0.07 & -0.12 & 0.15 & 0.24 & 0.12 & 0.09 & -0.05 & 0.05 & \\
10. Payment for vaccine & 0.40 & 0.25 & 0.10 & 0.29 & -0.26 & -0.26 & -0.23 & -0.24 & $\mathbf{0 . 5 0}$ \\
\hline
\end{tabular}

Note. Correlations in bold are between items sharing a topic.

Correlations between honesty-humility, extraversion, agreeableness, and COVID-19 attitudes are in Table 5. The initial rows display inter-correlations between traits; these are Pearson correlations. The remaining rows are polyserial correlations. The sample size for HEXACO traits is larger and readers should note the implication for statistical power when reviewing this table and the following table.

Participants who were high in honesty-humility and agreeableness (both types) were less likely to get a vaccine, but these correlations were weak $(-.18,-.19$, and -.17$)$. The theoretical reason for these correlations is difficult to discern. However, some scholars have suggested that the honesty-humility should be reverse scored and construed as a negative factor (Diebels, Leary, \& Chon, 2018) and even the scale creators have emphasized that low scorers merit more attention (Lee \& Ashton, 2013). Low levels of honesty-humility indicate dispositional selfishness and entitlement (Diebels et al., 2018). The negative correlations could indicate that people who are more entitled are more interested in self-preservation. Modal interest in the vaccine was also high - the highest value was the mode — so the small size of the correlation may be an artifact of the compressed range. Given a better measure, one might find a stronger correlation.

Participants who were high in Big-Five extraversion were more likely to get a vaccine (.26), suggesting a tenuous connection between approach motivation and vaccination, but there no corresponding correlation for HEXACO extraversion (.07). The difference between Big-Five and HEXACO extraversion may be pertinent, but the inconsistency could also indicate an effect 
produced by random chance. Perceived risk of infection was positively associated with both HEXACO extraversion (.16) and Big Five extraversion (.15). These modest correlations suggest that extraverted people may realize their socializing increases their risk of infection and that less extraverted people realize their infrequent socializing protects them from infection.

Respondents who were high in Big Five agreeableness were more likely to perceive COVID-19 as severe (.24). The reason for this correlation is unclear and there was no corresponding correlation for HEXACO agreeableness (.07). A possible reason is that trust, a facet of Big Five agreeableness, drives people to believe scientific leaders but this hypothesis is speculative. Trust is not a facet of HEXACO agreeableness.

Table 5. Pearson and polyserial correlations between honesty-humility, extraversion, agreeableness, and COVID-19 attitudes

\begin{tabular}{lrrrrr}
\hline & $\mathrm{H}-\mathrm{H}$ & $\mathrm{Ag}(\mathrm{HEX})$ & $\mathrm{Ag}(\mathrm{B} 5)$ & $\mathrm{Ex}(\mathrm{HEX})$ & $\mathrm{Ex}(\mathrm{B} 5)$ \\
\hline Traits & & & & & \\
Agreeableness (HEXACO) & 0.37 & & & & \\
Agreeableness (B5) & 0.29 & 0.50 & & & \\
Extraversion (HEXACO) & -0.10 & 0.08 & 0.01 & & \\
Extraversion (B5) & -0.14 & -0.29 & 0.05 & 0.81 & \\
Attitudes & & & & & \\
U.S. Preparedness & 0.11 & 0.14 & 0.12 & -0.03 & -0.07 \\
European Preparedness & 0.07 & 0.11 & 0.09 & 0.12 & 0.07 \\
Risk of infection & -0.17 & -0.06 & -0.10 & 0.16 & 0.15 \\
Good possibility of infection & -0.12 & 0.01 & -0.11 & 0.04 & 0.05 \\
COVID is severe & 0.01 & 0.07 & 0.24 & 0.10 & 0.05 \\
COVID deadlier than measles & -0.05 & 0.02 & 0.10 & 0.11 & 0.03 \\
Hazardous to share & & & & & \\
class/workspace & -0.06 & 0.04 & -0.11 & 0.18 & -0.07 \\
Hazardous to share airplane row & -0.09 & -0.10 & -0.01 & -0.08 & -0.05 \\
Likelihood of getting vaccine & -0.18 & -0.19 & -0.17 & 0.07 & 0.26 \\
Max. payment for vaccine & -0.01 & -0.01 & 0.04 & 0.09 & 0.16 \\
\hline N & 172 & 172 & 100 & 172 & 100 \\
\hline
\end{tabular}

Note. Trait-trait correlations are Pearson correlations. All other correlations are polyserial correlations. Saturation in the lower part of the table indicates absolute value. $\mathrm{H}-\mathrm{H}=$ honestyhumility. 
Table 6. Pearson and polyserial correlations between conscientiousness, openness to experience, emotionality, and COVID-19 attitudes

\begin{tabular}{lrrrrrr}
\hline & $\mathrm{Co}(\mathrm{HEX})$ & $\mathrm{Co}(\mathrm{B} 5)$ & $\mathrm{Op}(\mathrm{HEX})$ & $\mathrm{Op}(\mathrm{B} 5)$ & $\mathrm{Em}(\mathrm{HEX})$ & $\mathrm{Em}(\mathrm{B} 5)$ \\
\hline Traits & & & & & & \\
Conscientiousness (B5) & 0.83 & & & & & \\
Openness (HEXACO) & 0.12 & -0.07 & & & & \\
Openness (B5) & 0.03 & 0.00 & 0.75 & & & \\
Emotionality (HEXACO) & 0.15 & -0.01 & -0.02 & 0.01 & & \\
Neg. Emotionality (B5) & -0.22 & -0.36 & 0.02 & -0.05 & 0.62 & \\
Attitudes & & & & & & \\
U.S. Preparedness & 0.14 & 0.32 & -0.21 & -0.15 & -0.04 & -0.11 \\
European Preparedness & 0.16 & 0.26 & 0.04 & 0.01 & -0.02 & -0.15 \\
Risk of infection & -0.11 & -0.07 & 0.05 & 0.04 & 0.00 & -0.02 \\
Good possibility of infection & -0.23 & -0.19 & -0.02 & -0.10 & 0.08 & 0.09 \\
COVID is severe & 0.10 & 0.07 & 0.09 & 0.04 & 0.14 & 0.09 \\
COVID deadlier than measles & 0.10 & 0.09 & -0.03 & -0.01 & -0.05 & -0.06 \\
Hazardous to share & & & & & & \\
class/workspace & -0.08 & -0.18 & -0.15 & -0.19 & -0.03 & -0.05 \\
Hazardous to share airplane row & -0.12 & -0.04 & -0.22 & -0.09 & 0.05 & 0.00 \\
Likelihood of getting vaccine & 0.03 & -0.10 & -0.06 & 0.01 & 0.02 & 0.06 \\
Max. payment for vaccine & 0.01 & 0.01 & -0.06 & 0.03 & 0.05 & -0.14 \\
\hline N & 172 & 100 & 172 & 100 & 172 & 100 \\
\hline
\end{tabular}

Note. Trait-trait correlations are Pearson correlations. All other correlations are polyserial correlations. Saturation indicates absolute value. 
Both types of conscientiousness predicted the tendency to have more faith in U.S. government preparedness for future outbreaks $(.14 ; .32)$, whereas both types of openness predicted the tendency to have less faith in U.S. preparedness (-.21, -.15). People who are conscientious are likely to be conservative and thus may trust the current conservative president of the U.S., whereas people who are open are likely to be liberal and have less confidence in this president (Carney, Jost, Gosling, \& Potter, 2008). Thus, political ideology could explain this pattern of associations with U.S. preparedness. It is unclear why conscientious respondents were also more confident in European governments $(.16 ; .26)$.

Correlations between conscientiousness, openness to experience, emotionality, and COVID-19 attitudes are in Table 6. The liberality of respondents who are high in openness could also explain another association. High-openness respondents were less likely to consider it hazardous to share space in a room $(-.15 ;-.19)$ or airplane $(-.22 ;-.09)$ with a person who might be infected.

Conscientious respondents also estimated they were likely to be infected in the future ($.23 ;-.19)$, perhaps due to their self-awareness - conscientious people are habitually diligent and cautious, and therefore may consistently follow health guidelines (cf. Molloy, O'Carroll, \& Ferguson, 2013). Surprisingly, conscientious people were also less wary of being in the vicinity of people who may be carrying COVID-19, but these correlations were mostly weak, ranging from -.08 to -.18 .

People who are high in emotionality tend be fearful and anxious. Accordingly, Big Five negative emotionality predicted less faith in U.S. preparedness (-.11) and European preparedness (-.15), and HEXACO emotionality predicted the perceived severity of COVID-19 (.14) (cf. Kroencke et al., 2020). These correlations were not strong. Surprisingly, respondents who were high in Big Five negative emotionality were less willing to pay a large amount for the vaccine (.14). Again, this correlation may be too weak to carry meaning. The question could have also tapped into the ideal amount that should be charged for the vaccine, and thus a lower amount could indicate that the respondent believed that the government should subsidize the vaccine.

To supplement the main analysis, I analyzed whether recent international travel experience was associated with COVID-19 attitudes (cf. Painter et al., 2017). As noted earlier (see Table 1), international travel experience was measured using a question about how many international flights the participant had taken in the past 12 months, answered on a 5-point scale 
with a minimum of zero and a maximum of 7 or more. There were three findings of note (see Table 7). People with more travel experience were more likely to say they had a good possibility of infection (.16), less likely to say that sharing a class or workspace was risky (-.23), and less likely to say that sitting next to someone on an airplane from an affected country was risky ($.19)$.

Table 7. Polychoric correlations between number of recent international flights and attitudes

\begin{tabular}{lc}
\hline & $\begin{array}{c}\text { Intl. } \\
\text { Travel }\end{array}$ \\
\hline U.S. Preparedness & -0.01 \\
European Preparedness & 0.10 \\
Risk of infection & 0.10 \\
Good possibility of infection & 0.16 \\
COVID is severe & 0.03 \\
COVID deadlier than measles & -0.04 \\
Hazardous to share class/workspace & -0.23 \\
Hazardous to share airplane row & -0.19 \\
Likelihood of getting vaccine & 0.10 \\
Max. payment for vaccine & 0.01 \\
\hline
\end{tabular}

\section{Discussion}

The current study explored whether personality traits predicted a range of attitudes toward COVID-19, personal risk, and public health. The study was not motivated by specific hypotheses but rather conducted to capitalize on the brief span of time when COVID-19 was the most salient public concern. At an absolute level, the study suggests that over half of all college students do not believe that U.S. and European governments are well prepared for future outbreaks. Confidence in the U.S. government was particularly low. This lack of confidence is not unique to this student sample - at least half of Americans were disappointed in the president's handling of the COVID-19 pandemic (Skelley, 2020).

In contrast, the strong interest in vaccines suggests that students' confidence in the medical community was high. The students in the sample were majoring in biomedical engineering, which was a confound here. These findings may not more relevant to political 
psychology, especially for researchers studying the youth vote and the medical-professional vote in the 2020 election.

Personality traits were the focus of this study. Most polyserial correlations between traits and attitudes were weak, and there was not a single correlation with an absolute value greater than .5. Nevertheless, many findings were similar across corresponding HEXACO and Big Five traits, suggesting some robust links between traits and COVID-19 attitudes. (Readers who are unfamiliar with these systems should note that traits with the same name across HEXACO and the Big Five are not identical but do have some correspondence.) One similar finding across the two trait systems was that conscientious students had more faith and high-openness students had less faith in U.S. preparedness. The liberality of high-openness participants was also evinced. They were less suspicious of people who had or were likely exposed to COVID-19. If these are indeed politically phenomena, these associations is malleable and may strengthen or weaken in the future as elite messaging evolves (Federico \& Malka, 2018). Elite messaging can changeand even reverse - the conventional associations between psychological dispositions and outcomes. Future researchers may compare their findings with the current study to examine whether trait-attitude associations are moving in sync with political messaging. Measuring political ideology and party affiliation would also be useful. We did not measure them in the current study.

The association between high openness and low risk aversion suggests that highopenness people may be incautious during pandemics, although these data do not permit an estimation of whether this relative difference increases real disease risk. These findings should be replicated with representative samples and behavioral measures. However, if this finding turns out to be robust, one implication is public health agencies may find it efficient and effective to focus their prevention messaging on people in high-risk populations who are high in openness; messaging could be packaged with products or displayed at sites that attract high-openness people,

The results also suggest that conscientious people estimate themselves as having a lower risk of infection. If this finding is replicable, it may be worthwhile to discern between two possible explanation. The first is that conscientious people are aware of the protective effects of conscientiousness. The second is that conscientious people have more trust in conservative governments. 
The results of the study may be most useful to social psychologists who use Bayesian analysis. They can use the correlations in this study (and similar studies) to set Bayesian priors. Given the unrepresentative sample, the results of the current study are not as generalizable as a weighted population survey, but the correlations carry some informational value, especially because the traits in this study were measured using long questionnaires. Most large polls conducted by sociologists and political sciences use much shorter trait scales, thereby sacrificing quality of measurement. 


\section{References}

Abdelrahman, M. (2020). Personality traits, risk perception and protective behaviors During COVID-19. https://doi.org/10.31234/osf.io/6g7kh

Carney, D. R., Jost, J. T., Gosling, S. D., \& Potter, J. (2008). The secret lives of liberals and conservatives: Personality profiles, interaction styles, and the things they leave behind. Political Psychology, 29(6), 807-840. https://doi.org/10.1111/j.1467-9221.2008.00668.x

Diebels, K. J., Leary, M. R., \& Chon, D. (2018). Individual differences in selfishness as a major dimension of personality: A reinterpretation of the sixth personality factor. Review of General Psychology, 22(4), 367-376. https://doi.org/10.1037/gpr0000155

Federico, C. M., \& Malka, A. (2018). The Contingent, Contextual Nature of the Relationship Between Needs for Security and Certainty and Political Preferences: Evidence and Implications. Political Psychology, 39, 3-48. https://doi.org/10.1111/pops.12477

Flesia, L., Fietta, V., Colicino, E., Segatto, B., \& Monaro, M. (2020). Stable psychological traits predict perceived stress related to the COVID-19 outbreak. https://doi.org/10.31234/osf.io/yb2h8

Healthy Minds Network. (2016). The Healthy Minds study. Retrieved from http://www.healthymindsnetwork.org

Kondo, M., Hoshi, S., \& Okubo, I. (2009). Does subsidy work? Price elasticity of demand for influenza vaccination among the elderly in Japan. Health Policy, 91(3), 269-276. https://doi.org/10.1016/j.healthpol.2008.12.014

Kraus, M. W., Piff, P. K., Mendoza-Denton, R., Rheinschmidt, M. L., \& Keltner, D. (2012). Social class, solipsism, and contextualism: How the rich are different from the poor. Psychological Review, 119(3), 546-572. https://doi.org/10.1037/a0028756

Kroencke, L., Geukes, K., Utesch, T., Kuper, N., \& Back, M. (2020). Neuroticism and emotional risk During the Covid-19 pandemic. https://doi.org/10.31234/osf.io/8c6nh

Lee, K., \& Ashton, M. C. (2013). The H factor of personality: Why some people are manipulative, self-entitled, materialistic, and exploitive and why it matters for everyone. Waterloo, Canada: Wilfrid Laurier Univ. Press.

Lee, K., \& Ashton, M. C. (2018). Psychometric properties of the HEXACO-100. Assessment, 25(5), 543-556. https://doi.org/10.1177/1073191116659134 
Martin, C. C. (2020). How education did (And did not) accentuate partisan differences during the Ebola outbreak of 2014-15. Journal of Social and Political Psychology, 8(1), 108-131. https://doi.org/10.5964/jspp.v8i1.1072

Molloy, G. J., O’Carroll, R. E., \& Ferguson, E. (2013). Conscientiousness and medication adherence: A meta-analysis. Annals of Behavioral Medicine, 1-10. https://doi.org/10.1007/s12160-013-9524-4

Painter, J. E., DiClemente, R. J., \& von Fricken, M. E. (2017). Interest in an Ebola vaccine among a U.S. national sample during the height of the 2014-2016 Ebola outbreak in West Africa. Vaccine, 35(4), 508-512. https://doi.org/10.1016/j.vaccine.2016.11.093

Skelley, G. (2020). Americans are largely unimpressed with Trump's handling of the coronavirus pandemic. Retrieved May 19, 2020, from https://fivethirtyeight.com/features/americans-are-largely-unimpressed-with-trumpshandling-of-the-coronavirus-pandemic/

Soto, C. J., \& John, O. P. (2017). The next Big Five Inventory (BFI-2): Developing and assessing a hierarchical model with 15 facets to enhance bandwidth, fidelity, and predictive power. Journal of Personality and Social Psychology, 113(1), 117-143. https://doi.org/10.1037/pspp0000096

Turiano, N. A., Pitzer, L., Armour, C., Karlamangla, A., Ryff, C. D., \& Mroczek, D. K. (2012). Personality Trait Level and Change as Predictors of Health Outcomes: Findings From a National Study of Americans (MIDUS). The Journals of Gerontology Series B:

Psychological Sciences and Social Sciences, 67B(1), 4-12. https://doi.org/10.1093/geronb/gbr072 


\section{Appendix}

These questions are adapted from the Emory University 2015 Ebola Survey. For related publications see:

Martin. (2020). How Education Did (and Did Not) Accentuate Partisan Differences During the Ebola Outbreak of 2014-15 https://jspp.psychopen.eu/article/view/1072/html

Painter et al. (2017a). Willingness to pay for an Ebola vaccine during the 2014-2016 ebola outbreak in West Africa: Results from a U.S. National sample https://www.tandfonline.com/doi/full/10.1080/21645515.2018.1423928

Painter et al. (2017b). Interest in an Ebola vaccine among a U.S. national sample during the height of the 2014-2016 Ebola outbreak in West Africa

https://www-sciencedirect-

com.prx.library.gatech.edu/science/article/pii/S0264410X1631204X

\section{Travel Experience}

How many international flights have you taken in the past 12 months?

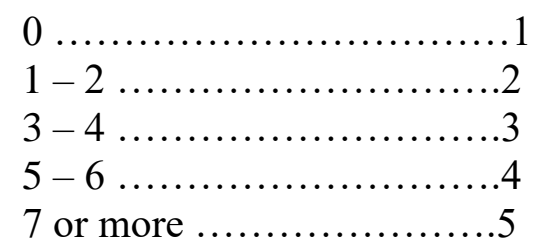

Have you traveled to Italy, Iran, South Korea, or China in the past 2 months?

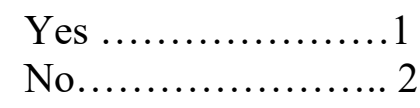

\section{Preparedness Block}

These items were answered on a four-point scale: not prepared, somewhat prepared, fairly prepared, and very prepared.

How prepared do you think the U.S. government is when it comes to containing the spread of potential future global health epidemics within their geographic areas?

How prepared do you think European national governments are when it comes to containing the spread of potential future global health epidemics within their geographic areas?

\section{Fear of Infection Block}


These items were answered on a four-point scale: strongly disagree, disagree, agree, strongly agree.

My risk of getting infected with COVID-19 (Coronavirus) is high.

There is a good possibility that I will get infected with COVID-19 (Coronavirus).

\section{Severity Block}

These items were answered on a four-point scale: strongly disagree, disagree, agree, strongly agree.

COVID-19 (Coronavirus) is a severe disease.

COVID-19 (Coronavirus) is more deadly than the measles.

\section{Suspicion Block}

These items were answered on a four-point scale: strongly disagree, disagree, agree, strongly agree.

I would not want to be in the same classroom or work space with a person who had previously been diagnosed with COVID-19 (Coronavirus).

If I sit next to someone on the airplane from a country where COVID-19 (Coronavirus) has been widespread, there is a strong likelihood that I would get infected with COVID-19.

\section{Vaccine Block}

For this set of questions, assume there was an FDA approved vaccine to prevent COVID-19. How likely would you be to get a vaccine that would prevent you from getting COVID-19?

Not likely

Somewhat likely

Fairly likely

Very likely

How much would you pay out of pocket for a COVID-19 vaccine if your health insurance did not cover the cost of the vaccine?

$\$ 0$ (I would not pay for a COVID vaccine)............1

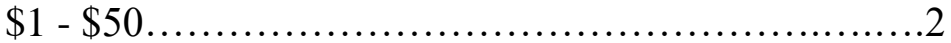

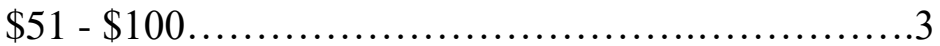




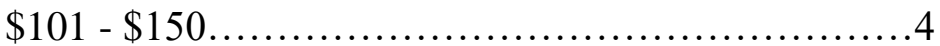

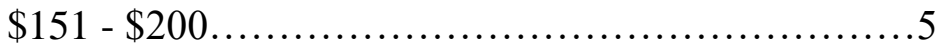

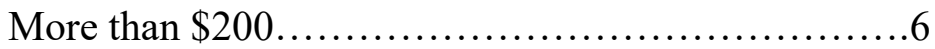

